\title{
Evolution of Disks in the Course of Star Formation
}

\author{
Masahiko HAYASHI \\ Department of Astronomy, The University of Tokyo, Bunkyo-ku, Tokyo 113, \\ Japan
}

\begin{abstract}
Observations of circumstellar disks with Nobeyama Millimeter Array (NMA) are presented for the following two topics. The first one is on the continued NMA survey for 13 complete samples of protostar candidates associated with Taurus molecular cloud. The observation confirmed the previous result that protostar candidates do not have detectable $3 \mathrm{~mm}$ continuum emission except for the two sources L1551-IRS5 and IRAS 04365+2535. This sets the upper limit to the circumstellar disk mass to be $\sim 0.03 \mathrm{M}_{\odot}$ for the protostar candidates. The disk mass for protostar candidates tends to be smaller than that around young $\mathrm{T}$ Tauri stars, suggesting that it may increase in the course of evolution from protostars into $\mathrm{T}$ Tauri stars. The second topic is on the detection of CO $(J=1-0)$ emission toward GG Tau. The observations with the 45-m telescope and with NMA show strong evidence of the $\mathrm{CO}$ emission arising from a rotating disk with its size significantly extended with respect to the dust disk. Depletion of CO gas in the GG Tau disk is discussed.
\end{abstract}

\section{Introduction}

Observations in millimeter, infrared, and optical wavelengths in 1980's have revealed evidence of circumstellar dust disks around T Tauri stars (Rucinski 1985; Adams, Lada, \& Shu 1987; Appenzeller et al. 1984; Edwards et al. 1987; Bertout 1989). Such disks are believed to be protoplanetary disks, since their mass and size, $\sim 0.01 \mathrm{M}_{\odot}$ and $\lesssim 100 \mathrm{AU}$, respectively (Beckwith et al. 1990), are similar to those of the minimum mass solar nebula (Hayashi, Nakazawa, \& Nakagawa 1985).

Evolutionary studies of circumstellar disks based on statistical analyses play major roles in understanding the formation process of planetary systems. Beckwith et al. (1990) made a $1.3 \mathrm{~mm}$ survey observation for dust disks toward $86 \mathrm{~T}$ Tauri stars associated with Taurus molecular cloud. They found that the disk mass does not decrease in $10^{7} \mathrm{yr}$, suggesting that the outer part of disks are stable during at least $10^{7} \mathrm{yr}$. The inner part of disks may, however, disappear at a time scale of $3 \times 10^{6} \mathrm{yr}$, as was suggested by the rapid decrease in near- and midinfrared excess emissions toward T Tauri stars with their ages of $\sim 3 \times 10^{6} \mathrm{yr}$ (Strom et al. 1989; Skrutskie et al. 1990). While the dissipation process of disks can thus be traced with the aid of age information of $T$ Tauri stars, their formation process may be observed in the course of evolution from embedded protostars into $T$ Tauri stars. For this purpose, it is important to compare the circumstellar mass distribution between protostellar objects and $\mathrm{T}$ Tauri stars. I will introduce, as a first topic of this article, a survey observation toward 13 complete samples of IRAS selected protostar candidates in Taurus. The results suggest that protostar candidates, on average, do not have circumstellar mass distribution as highly concentrated as $\mathrm{T}$ Tauri stars.

Another important topic that I will describe here is the gas associated with protoplanetary disks. Although great efforts have so far been made to detect gas component in protoplanetary 
disks, very few sources such as HL Tau show evidence of gas disks with $\sim 100 \mathrm{AU}$ in size (Sargent and Beckwith 1991). Detection of gas emission from circumstellar disks is difficult not only because line emissions from such disks are weak but they are contaminated by strong background and/or foreground emissions arising from ambient molecular gas. Suppose observing the $\mathrm{CO}(J=1-0)$ emission from a gas disk of $\sim 100 \mathrm{AU}$ in radius at the distance of the Taurus cloud with the $45-\mathrm{m}$ telescope, we would obtain an antenna temperature of $\lesssim 50 \mathrm{mK}$ (Omodaka et al. 1992); it must be impossible to detect the line emission with 10-m class telescopes because the intensity becomes more than 10 times less than this. A weak emission line of $\sim 50 \mathrm{mK}$ can, however, be detected if there would be no background or foreground emission typically of $1-10 \mathrm{~K}$ in the Taurus cloud. It is therefore crucial to find T Tauri stars with very low background or foreground $\mathrm{CO}$ emission, although it is rare to find such $T$ Tauri stars. GG Tau is a good example of no ambient ${ }^{12} \mathrm{CO}$ emission when observed with the FCRAO 14-m telescope (Skrutskie et al. 1993). One may consider that emission lines of molecules with high critical density would be less contaminated by the foreground and/or background emission, so that those lines would be useful for detecting gas in disks. Such molecular lines may be weaker than the $\mathrm{CO}$ lines, because molecules with high condensation temperature are depleted in circumstellar disks as was the case for CS (Blake et al. 1992).

\section{Difference in Circumstellar Mass Distribution between Protostars and T Tauri Stars}

Ohashi et al. (presented in this colloquium) made a survey observation of protostar candidates and young $\mathrm{T}$ Tauri stars in the $3 \mathrm{~mm}$ continuum and $\mathrm{CS}(J=2-1)$ line emissions with NMA. Protostar candidates are the IRAS sources satisfying the following conditions: (1) they are not visible on Palomar Observatory Sky Survey, and (2) they show IRAS colors cooler than those of T Tauri stars (Beichman et al. 1986). Details of the source selection were described by Ohashi (1991), who selected 13 unambiguous candidates for protostars located in the Taurus molecular cloud. They limited their targets only for those sources associated with Taurus molecular cloud. This is because the mass of newly formed stars is within a narrow range (Cohen \& Kuhi 1979), so that evolutionary effects may be recognized much easier than in other molecular clouds with stars of wider mass range. After the initial results of the survey for 5 protostar candidates and $6 \mathrm{~T}$ Tauri stars were reported (Ohashi et al. 1991), they continued and finished observations of the 13 complete samples of protostar candidates.

Evidence of circumstellar disks around protostar candidates is mentioned below. The evidence is, however, much weaker than that around T Tauri stars; we still do not know the physical properties of disks around protostar candidates. Firstly, Myers et al. (1987) derived a visual extinction of $\lesssim 100 \mathrm{mag}$ toward the central light sources of protostar candidates. Since this value was much smaller than that predicted from simple spherical models for protostellar envelopes, namely $10^{3}-10^{4} \mathrm{mag}$, they claimed that the mass distribution around protostar candidates might not be spherical but probably be disk like within a radius of $\sim 100 \mathrm{AU}$ from the central sources. Secondly, phenomenological model fits to the spectral energy distribution of protostellar candidates show shortage at the midinfrared wavelength unless extra emissions from substellar temperature material are taken into account. The extra emissions may be originated from circumstellar disks (Adams, Lada, and Shu 1987). Thirdly Cabrit \& André (1991) found that low mass protostellar candidates with large $1.3 \mathrm{~mm}$ continuum fluxes tend to have well-developed molecular outflows, suggesting that strong outflows are driven by massive accretion disks. This also indicates the presence of disks around protostar candidates, although the actual disk mass around protostar candidates are still unknown.

Interferometric observations are essential to find out the mass distribution at a scale of $\lesssim 1,000 \mathrm{AU}$, not only because of their high angular resolutions but because of their sensitivity 
Table 1 Results of the NMA survey observations

\begin{tabular}{|c|c|c|c|c|c|c|c|}
\hline \multirow{2}{*}{$\begin{array}{l}\begin{array}{l}\text { Observed } \\
\text { Sources }\end{array} \\
\text { L1489 }\end{array}$} & \multirow{2}{*}{$\frac{\begin{array}{c}\text { Optical } \\
\text { Appesance }\end{array}}{\text { invisible }}$} & \multicolumn{2}{|c|}{$\begin{array}{r}\operatorname{CS}(=2-1) \\
\operatorname{lgs}\left(J \mathrm{kms}^{-1)} \operatorname{Size}(\mathrm{AU})^{p}\right.\end{array}$} & \multirow{2}{*}{$\frac{M\left(M_{\odot}\right)}{1.1 \times 10^{-1}}$} & \multicolumn{3}{|c|}{ 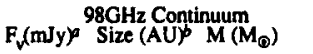 } \\
\hline & & $5.6 \pm 0.32$ & $2000 \times 1700$ & & $<9.6$ & & $<2.2 \times 10^{-2}$ \\
\hline $\begin{array}{l}04108+2803 \\
04154+2823\end{array}$ & $\begin{array}{l}\text { invisible } \\
\text { invisible }\end{array}$ & $\begin{array}{l}1.4 \pm 0.32 \\
<0.44\end{array}$ & $2700 \times 1200$ & $2.6 \times 10^{-2}$ & $\begin{array}{l}<21 \\
<19\end{array}$ & & $\begin{array}{l}<4.7 \times 10^{-2} \\
<4.4 \times 10^{-2}\end{array}$ \\
\hline $04169+2702$ & invisible & $2.6 \pm 0.32$ & $\begin{array}{l}1600 \times 1500^{d} \\
2000 \times 1200^{d}\end{array}$ & $4.9 \times 10^{-2}$ & $<14$ & & $<3.2 \times 10^{-2}$ \\
\hline $04191+1523$ & invisible & $0.80 \pm 0.085$ & $1400 \times 1400$ & $1.5 \times 10^{-2}$ & $<9,0$ & & $<2.1 \times 10^{-2}$ \\
\hline $04239+2436$ & invisible & 0.066 & $2400 \times 2100$ & $2.6 \times 10^{-2}$ & $<12$ & & $<3.2 \times 10^{-2}$ \\
\hline $04248+2612$ & invisible & $1.7 \pm 0.17$ & $2400 \times 2100$ & $4.3 \times 10^{-2}$ & $<12$ & & $<3.4 \times 10^{-2}$ \\
\hline $\begin{array}{l}04295+2251 \\
\text { L1551-IRS5 }\end{array}$ & $\begin{array}{l}\text { invisible } \\
\text { invisible }\end{array}$ & & $2300 \times 1600$ & 2.8 & $\underset{131 \pm 4.0}{<1}$ & $\times 360$ & $\begin{array}{l}<2.8 \times 10^{-2} \\
7.0 \times 10^{-2}\end{array}$ \\
\hline $04325+2402$ & invisible & $0.85 \pm 0.080$ & $1800 \times 1400$ & $1.6 \times 10^{-1}$ & $<9.2$ & & $<2.1 \times 10^{-2}$ \\
\hline $04361+2547$ & invisible & $1.4 \pm 0.32$ & $\begin{array}{l}2100 \times 1500^{d} \\
2100 \times 1500^{4}\end{array}$ & $2.6 \times 10^{-2}$ & $<14$ & & $<3.2 \times 10^{-2}$ \\
\hline $\begin{array}{l}04365+2535 \\
\text { L1527 }\end{array}$ & $\begin{array}{l}\text { invisible } \\
\text { invisible }\end{array}$ & $\begin{array}{c}2.5 \pm 0.095 \\
11 \pm 0.32\end{array}$ & $\begin{array}{l}1600 \times 1600 \\
2600 \times 2000\end{array}$ & $\begin{array}{l}4.6 \times 10^{-2} \\
2.1 \times 10^{-1}\end{array}$ & $\begin{array}{c}30 \pm 3.8 \\
<11\end{array}$ & $<940 \times 700$ & $\begin{array}{l}6.8 \times 10^{-2} \\
<2.5 \times 10^{-2}\end{array}$ \\
\hline $\begin{array}{l}\text { FS Tau } \\
\text { T Tau }\end{array}$ & $\begin{array}{l}\text { visible } \\
\text { visible }\end{array}$ & $\begin{array}{l}<0.34 \\
<0.60\end{array}$ & & & $\begin{array}{c}<11 \\
56 \pm 10\end{array}$ & $<550 \times 420$ & $\begin{array}{l}<4.1 \times 10^{-2} \\
1.0 \times 10^{-1}\end{array}$ \\
\hline DG Tau & visible & $1.4 \pm 0.32$ & 1600 & $2.6 \times 10^{-2}$ & $57 \pm 4.0$ & $<8000 \times 770$ & $1.0 \times 10^{-1}$ \\
\hline HL Tau & & $1.5 \pm 0.32$ & $1200 \times 850$ & $2.8 \times 10^{-2}$ & $74 \pm 4.0$ & $<530 \times 420$ & $1.5 \times 10^{-1}$ \\
\hline GG Tau & visible & $<0.44$ & & & $41 \pm 4,0$ & $<780 \times 670$ & $1.6 \times 10^{-1}$ \\
\hline DL Tau & visible & $<0.37$ & & & $23 \pm 4.0$ & $<1470 \times 880$ & $01.0 \times 10^{-1}$ \\
\hline
\end{tabular}

apper limit values are 30 and errors are 10

b FWH size

c Gas mass was derived under the assumption of optically thin CS emission

d Two spatially separate components are identified

only to the structures less than their resolving-out limits. With Nobeyama Millimeter Array, we can achieve an angular resolution of $\sim 2^{\prime \prime}$, corresponding to the linear scale of $\sim 300 \mathrm{AU}$ at a distance to the Taurus cloud. On the other hand, spatial structures with angular extents larger than $40^{\prime \prime}(\sim 5,000 \mathrm{AU})$ are resolved out at $100 \mathrm{GHz}$, since the minimum baseline length of NMA is $15 \mathrm{~m}$. Hence we can probe only the very inner part of protostellar envelopes without being affected by a large amount of matter extended around them as associated dense cores (Myers, Benson, and Linke 1983; Beichman et al. 1986).

Table 1 summarizes the observations so far made toward 13 complete samples of protostar candidates and 6 young $\mathrm{T}$ Tauri stars. The fifth column shows the gas mass contained in each CS emitting area derived under the assumption of optically thin CS emission. This mass actually gives a lower limit to the gas mass because the CS emission is optically thick. The last column lists the total gas mass within the beam size derived from the dust continuum flux under the assumption that the gas to dust mass ratio is 100 , that the power law index of grain emissivity is 1 , and that the temperature of the $3 \mathrm{~mm}$ emitting regions is $30 \mathrm{~K}$ for most cases. Details of the mass derivation are described in Ohashi et al. (1991).

The CS emission is extended over 1,000-2,000 AU around or near the central objects. Protostar candidates are associated with extended envelopes of $\sim 10,000 \mathrm{AU}$ in radius. Hence the extended CS emission arises from very inner part of such envelopes; most of the emission contained in outer envelopes was completely resolved out by the present interferometric observations. The CS emission does not spatially coincide with stellar positions for the 3 protostar candidates $04108+2803,04248+2612$, and $04361+2547$. This is caused probably because the optically thick CS $(J=2-1)$ emission does not follow the actual mass distribution around those sources with bad positional coincidence. For the case of $2 \mathrm{~T}$ Tauri stars DG Tau and HL Tau detected in CS, the CS emission does not coincide with their stellar positions, either. Small visual extinctions (a few mag) toward these $T$ Tauri stars suggest that we would have detected the CS emission directly associated with the circumstellar disks around them without contamination from foreground or background CS emission, if the CS disks had a 


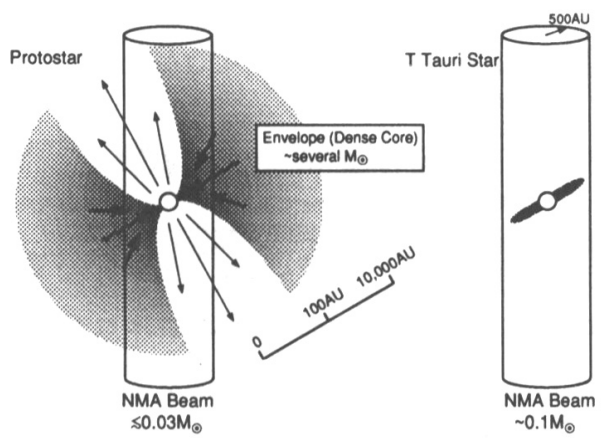

Figure 1 A schematic picture showing the difference in circumstellar mass distribution

size of $\sim 70 \mathrm{AU}$ or more. No detectable CS emissions directly associated with the stars implies that CS molecules may be depleted in their disks, as was suggested for HL Tau by Blake, van Dishoeck, \& Sargent (1992).

The $98 \mathrm{GHz}$ continuum emission was detected from 2 out of 13 protostar candidates, L1551-IRS5 and 04365+2535, and from 5 out of $6 \mathrm{~T}$ Tauri stars. The emission size was not resolved by the NMA beam of $\sim 500 \mathrm{AU}$ in radius; the continuum emission is consistent with arising from compact dust disks around these objects. It is remarkable in Table 1 that only two protostar candidates showed detectable continuum emission, whereas most of the $T$ Tauri stars were detected in the continuum. Because we selected $T$ Tauri stars with strong $1.3 \mathrm{~mm}$ and $100 \mu \mathrm{m}$ fluxes, it is reasonable that most of them also b. .2 strong $3 \mathrm{~mm}$ flux from dust disks; the high detectability of the continuum emission toward $T$ Tauri stars may be a selection effect. On the other hand, the small detectability toward protostar candidates are real since we observed all the protostar candidates selected under the definite criteria. The upper limit to the total mass within the beam size of $\sim 500 \mathrm{AU}$ is typically $0.03 \mathrm{M}_{\odot}$ for protostar candidates if we assume similar disk temperature for protostar candidates as for T Tauri stars.

The difference in the $98 \mathrm{GHz}$ continuum and CS line detectabilities between the two types of objects is attributable to the difference in circumstellar mass distribution between them, as is schematically illustrated in Figure 1. The protostar candidates tend to have large mass at 1,000-2,000 $\mathrm{AU}$ in radius or at larger radii, while they do not have so much mass within a radius of $500 \mathrm{AU}$ as $\mathrm{T}$ Tauri stars do. This may indicate that many protostars in Taurus have disk mass smaller than the present upper limit of $0.03 \mathrm{M}_{\odot}$. Such small mass in the protostellar phase is consistent with the relatively small luminosity of protostar candidates in Taurus and agrees well with current theories on steady formation of star/disk systems (Stahler, Shu, and Taam 1980; Nakamoto and Nakagawa 1993). The disk mass around protostars increases with the evolution of protostars into $\mathrm{T}$ Tauri stars.

\section{A Gas Disk associated with GG Tau}

Figure 2 shows the ${ }^{12} \mathrm{CO}(J=1-0)$ line profile detected toward GG Tau and the negative result for the $C^{18} \mathrm{O}(J=1-0)$ emission (Skrutskie et al. 1993). The ${ }^{12} \mathrm{CO}$ emission was detected with $\sim 0.4 \mathrm{~K}$ in antenna temperature, while it was not detected with the upper limit of $50 \mathrm{mK}$ with the FCRAO 14-m telescope, which has ten times larger beam solid angle than the 45$\mathrm{m}$ telescope. This means that the $\mathrm{CO}$ emission detected with the $45-\mathrm{m}$ telescope must be compact and be directly associated with GG Tau. The double peaked CO emission profile is well fitted by a disk model with $\sim 500$ AU in radius.

Figure 3 shows the channel maps of ${ }^{12} \mathrm{CO}(J=1-0)$ emission toward GG Tau observed with NMA (Kawabe et al. 1993). The CO maps show shift in the emission peak from east 

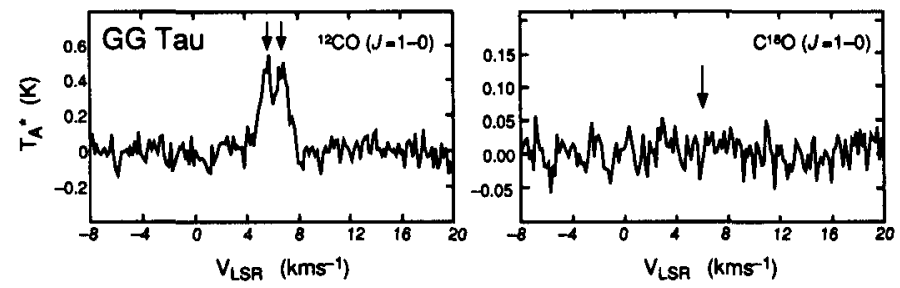

Figure $2{ }^{12} \mathrm{CO}$ and $\mathrm{C}^{18} \mathrm{O}$ data obtained with the 45 -m telescope (Skrutskie et al. 1993)

to west with increasing velocity from 5.7 to $7.3 \mathrm{kms}^{-1}$, being consistent with a rotating gas disk model with the radius and rotational velocity of $500 \mathrm{AU}$ and $0.8 \mathrm{kms}^{-1} / \sin i$, respectively. Integrating the channel maps over the emission velocity width, we obtain the total $\mathrm{CO}$ intensity map elongated in the northwest to southeast direction with the size of $10^{\prime \prime} \times 6^{\prime \prime}(1,400 \mathrm{AU} \times 840 \mathrm{AU})$. The integrated CO map shows the similar morphology and position angle to the IRAM $2.6 \mathrm{~mm}$ continuum map (Simon and Guilloteau 1993). Although the size of the CO emission is approximately twice as large as that of the continuum, this is natural because the $\mathrm{CO}$ emission arises from less dense outer regions compared to the continuum emission as a result of the higher mass emissivity for $\mathrm{CO}$ than for continuum.

The NMA map of ${ }^{12} \mathrm{CO}(J=1-0)$ agrees sufficiently well with the ${ }^{13} \mathrm{CO}(J=1-0)$ map obtained at OVRO (presented by Dr. A. Sargent in this colloquium). She suggested, however, that the actual gas disk would be elongated in the north-south direction based on the ${ }^{13} \mathrm{CO}$ $(J=2-1)$ observations at OVRO (see the discussion following this paper). For the purpose of understanding the gas disk around GG Tau, we must wait for further observations with higher sensitivities and spatial resolutions so that we can resolve the spatial and velocity structure of the gas emission associated with GG Tau (see Dutrey, Guilloteau, \& Simon 1994).

A very important conclusion derived from the negative result for the $\mathrm{C}^{18} \mathrm{O}$ line shown in Figure 2 is the depletion of $\mathrm{CO}$ gas with respect to dust. Integrating over the velocity width of $2 \mathrm{kms}^{-1}$, we can set the upper limit to the $\mathrm{C}^{18} \mathrm{O}$ intensity as $0.11 \mathrm{~K} \mathrm{kms}^{-1}$. This gives the total amount of $\mathrm{C}^{18} \mathrm{O}$ within the beam size $2,000 \mathrm{AU}$ of the $45-\mathrm{m}$ telescope to be less than $7 \times 10^{-9} \mathrm{M}_{\odot}$. The total mass of dust grains within the NMA beam size is $1.6 \times 10^{-3} \mathrm{M}_{\odot}$ as was in Table 1 (Note that the total mass of $0.16 \mathrm{M}_{\odot}$ in Table 1 was obtained by assuming the gas to dust mass ratio of 100 ). The ratio of $\mathrm{C}^{18} \mathrm{O}$ mass to the dust mass is hence $4 \times 10^{-6}$, which is one order of magnitude smaller than the ratio of $4 \times 10^{-5}$ calculated with nominal interstellar abundances. Hence the $\mathrm{CO}$ gas is significantly depleted with respect to dust.

It might be suggested that $\mathrm{C}^{18} \mathrm{O}$ was not detected as a result of beam dilution, but not as a result of $\mathrm{CO}$ depletion. If optically thick $\mathrm{C}^{18} \mathrm{O}$ gas would be concentrated within $\sim 70 \mathrm{AU}$ in radius, it would result in no detection of the $\mathrm{C}^{18} \mathrm{O}$ emission due to beam dilution. However the dust disk around GG Tau has been resolved with the IRAM interferometer to have the size of $500 \mathrm{AU} \times 700 \mathrm{AU}$ (Simon and Guilloteau 1993; Dutrey et al. 1994), so that the beam dilution is not the cause of the non-detection of $\mathrm{C}^{18} \mathrm{O}$, if the gas disk has similar size to the dust disk.

The depletion of $\mathrm{CO}$ gas with respect to dust means either of the following two cases or both. (1) The fractional abundance of $\mathrm{CO}$ relative to $\mathrm{H}_{2}$ in the circumstellar disk of GG Tau is at least 10 times smaller than the ordinary value in molecular clouds. This may suggest that most of CO molecules in the GG Tau disk are significantly depleted onto dust grains. (2) The gas to dust mass ratio is at least an order of magnitude smaller than the ordinary value of 100 for interstellar matter. This case may imply that most of the gas in the disk has already been dissipated. We need quantitative determination of the $\mathrm{H}_{2}$ abundance in T Tauri disks in order to distinguish the two cases. 


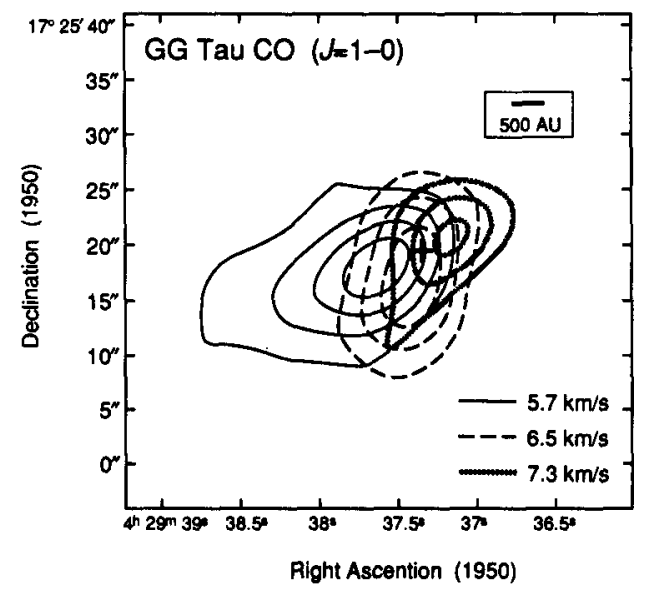

Figure 3 Map of $\mathrm{CO}(J=1-0)$ emission observed with NMA (Kawabe et al. 1993)

The observations presented here were carried out under collaborative works with Nagayoshi Ohashi, Ryohei Kawabe, Yasuo Fukui, Akira Mizuno, Toshikazu Ohnishi, Stephan Strom, Karen Strom, Mike Skrutskie, Ronald Snell, Suzan Edwards, Shoken Miyama, Toshihiro Omodaka, Yoshimi Kitamura, and Masato Ishiguro. I am grateful to Alan Tokunaga for valuable discussions.

\section{References}

Adams, F.C., Lada, C.J., \& Shu, F.H. 1987, ApJ, 312, 788

Appenzeller, I., Jankovics, I, \& Östreicher, R, 1984, AAp, 141, 108

Beichman, C.A., Myers, P.C., Emerson, J.P., Harris, S., Mathieu, R., Benson, P.J., \& Jennings, R.E. 1986, ApJ, 307, 337

Beckwith, S.V.W., Sargent, A.I., Chini, R.S., \& Güsten, R. 1990, AJ, 99, 924

Bertout, C. 1989, ARAA, 27, 351

Blake, G.A., van Dishoeck, E.F., \& Sargent, A.I. 1992, ApJ, 391, L99

Cabrit, S, \& André, P., 1991, ApJ, 379, L25

Cohen, M., \& Kuhi, L.V. 1979, ApJS, 41, 743

Dutrey, A., Guilloteau, S., \& Simon, M. 1994, AAp, in press

Hayashi, C., Nakazawa, K., \& Nakagawa, Y. 1985, in Protostars and Planets II, ed. D.C. Black \& M.S. Matthews (Tucson, University of Arizona Press), p.1100

Kawabe, R., Ishiguro, M., Omodaka, T., Kitamura, Y., and Miyama, S. 1993, ApJ., 404, L63 Rucinski, S.M. 1985, AJ, 90, 2321

Edwards, S.M. Cabrit, S., Strom, S.E., Heyer, I., Strom, K.M., \& Anderson,E. 1987, ApJ, 321,473

Myers, P.C., Linke, R., \& Benson, P. 1983, ApJ, 264, 517

Myers, P.C., Fuller, G.A., Mathieu, R.D., Beichman, C.A., Benson, P.J., Schild, R.E., \& Emerson, J.P., 1987, ApJ, 319, 340

Nakamoto, T. and Nakagawa, Y. 1993, submitted to ApJ

Ohashi, N., Kawabe, R., Hayashi, M., \& Ishiguro, M., 1991, AJ, 102, 2054

Ohashi N. 1991, thesis for DSc (Nagoya University)

Omodaka, T, Kitamura, Y., and Kawazoe, E., 1992, ApJ, 396, L87

Sargent, A.I., \& Beckwith, S.V.W., 1991, ApJ, 382, L31

Simon, M. and Guilloteau, S. 1992, ApJ, 397, L47.

Stahler, S.W., Shu, F.H, and Taam, R.E. 1980, ApJ, 241, 637

Strom, K.M, Strom, S.E., Edwards, S., Cabrit, S. \& Skrutskie, M.F. 1989, AJ, 1451

Skrutskie, M.F., Dutkevitch, D., Strom, S.E., Edwards, S., \& Strom, K.M. 1990, AJ, 99, 1187 


\section{DISCUSSION}

K. Stapelfeldt For your samples of the embedded objects and the T Tauri stars, have you made a comparison of the continuum detectability at $1.3 \mathrm{~mm}$ ?

M. Hayashi Some of the sources have been observed at $1.3 \mathrm{~mm}$, but not so many. Phillipe Andre - who was supposed to be here today - when you see his map in Rho Oph of the embedded objects, they usually have an extended structure. His maps of $T$ Tauri stars show compact structure.

K. Stapelfeldt One reason I think this question is important is that you expect the grain opacity will become less steep - the spectral index will go from 2 to 1 as you evolve in these early stages. The embedded sources may in fact have a different opacity spectral index than for the T Tauri stars, and that could make a factor of 10 difference in the amount of mass you can detect. It could explain the entire detectability difference you see between the two samples.

M. Hayashi It is usually said that the $B$ index of L1551 IRS5 is greater than 1 . When we compare 150 and $100 \mathrm{GHz}$ observations from the compact continuum source, we get a spectral index of 1 . So $I$ assume that $B$ must be 1 for all sources.

$\underline{K}$. Stapelfeldt It would be nice to know the value of $B$ for all your sources, if you could get it.

\section{T. Henning What are the selection criteria for IRAS sources?}

M. Hayashi Details of the source selection was discussed in Ohashi (1991). Among 1164 sources in the Taurus region listed in the IRAS point source catalog, he first selected 123 sources with significant detection at 3 or 4 wavelength bands including 25 and $60 \mu \mathrm{m}$. Excluding cataloged stars, galaxies, sources exhibiting colors of normal stars, and extended sources typical of cirrus emission, he then picked up 46 sources with protostellar characteristics. On the POSS atlas, 13 out of 46 have no optical counterparts, with the other 33 have visible counterparts, many of which were identified as T Tauri stars.

A. Sargent Let me respond to your contention that the 45-meter Nobeyama spectrum and the NMA results conflict with the studies of GG Tau at Owens valley. It is difficult to compare the $45-\mathrm{m} .15$ " resolution spectral line profile interpretation with our $5 " \times 3^{\prime 13} \mathrm{CO}(\mathrm{J}=2-1)$ images, which show elongated gas extending over $20 "$. It is clear that gas surrounds not just GG Tau, but also its companion system, GG Tau/c. Perhaps mapping around the central GG Tau position with the 45-m would resolve this discrepancy. However, it seems likely that the $\mathrm{CO}$ samples the ambient cloud 
rather than circumstellar material, since models of disks demonstrate that $\mathrm{CO}$, and even ${ }^{13} \mathrm{CO}$, emission in the direction of star-disk systems is very optically thick. The fact that our systemic velocity for the GG Tau material differs from the Nobeuama ${ }^{12} \mathrm{CO}$ peak velocity supports this view. Our ${ }^{13} \mathrm{CO}(\mathrm{J}=2-1)$ Owens Valley maps show clear evidence of a velocity gradient in our elongated gas structure along a N-S axis joining GG Tau and GG Tau/c. We see at blue-shifted velocities a weak distortion in the elongated structure, consistent with the blue shifted structure seen by Kawabe et al. in ${ }^{12} \mathrm{CO}$. We do not see the matching redshifted emission expected for rotation about a N-S axis. On the contrary, the bulk of the ${ }^{13} \mathrm{CO}(\mathrm{J}=2-1)$ emission (and the ${ }^{13} \mathrm{CO}(\mathrm{J}=1-0)$ emission $)$ is confined to the elongated N-S structure. Again we suggest that the optically thick $\mathrm{CO}$ emission icludes a large and confusing contribution from the ambient cloud.

M. Hayashi As I stated in the text, ${ }^{12} \mathrm{CO}(\mathrm{J}=1-0)$ emission toward GG Tau was not detected with an upper limit of $50 \mathrm{mK}$ by the FCRAO 14-m telescope which had a beam size 3 times larger than the 45-m telescope. NMA detected no significant emission except around GG Tau in spite of its wide field of view of $1^{\prime}(F W H M)$. These facts suggest that the ${ }^{12} \mathrm{CO}(\mathrm{J}=1-0)$ emission detected toward GG Tau is not much extended compared to the $15^{\prime \prime}$ beam of the 45-m telescope and is directly associated with the star/disk system. However we need further observations in order to clarify the true gas distribution atound GG Tau. Observations with the IRAM interferometer and with other molecular lines will solve the present discrepancy between your data and ours.

$\underline{N}$. Scoville It would be interesting to compare the disk axis in GG Tau as derived from optical polarization measurements with that of your mm line disk.

M. Hayashi Yes, polarization measurements are very important.

S.S. Hayashi About GG Tau disk, is there any comparison of continuum measurements to deduce dust disk properties, such as the morphology, spectral index, opacity? And any FIR/MIR high resolution measurements? (What is the "real" shape of the disk? Can it be delineated by molecular line observations, or dust continuum better?)

M. Hayashi You may refer to the papers by Beckwith et al.(1990) and Simon and Guilloteau (1992) as well as Kawabe et al.(1990) for the disk properties and its morphology. No one had directly observed the "real" shape of disks at any wavelength. Disks observed by molecular lines may be larger than those observed by dust continuum, because molecular line emissions are sensitive to much lower column density than the dust emission. 